\title{
Design of Ultra-wideband Low RCS Reflecting Screen Based on Phase Gradient Metasurface
}

\author{
Kefeng JI, Jun GAO, Xiangyu CAO, Jiangfeng HAN, Huanhuan YANG \\ Information and Navigation College of Air Force Engineering University, Xi'an, Shanxi, 710077, China \\ 806120093@qq.com
}

Submitted November 27, 2020 / Accepted March 12, 2021

\begin{abstract}
In order to realize full phase coverage of $360^{\circ}$ and abnormal reflection of incident wave, one kind of metasurface unit-cell with double diagonal opening cross petal and two types of one and two dimensional phase gradient metasurface with elements arranged on $6 \times 6$ array whose phase difference is $60^{\circ}$, were designed and constructed in this paper based on the abnormal reflection principle of phase gradient metasurface. By rotating the two phase gradient metasurfaces and arranging them in a spiral manner, three types of ultra-wideband low Radar Cross Section (RCS) reflecting screens with different unit configurations were devised. HFSS 14.0 was used for simulation experiment and the relative bandwidth of $R C S$ reduction above $10 \mathrm{~dB}$ is respectively $34.5 \%, 28.8 \%$ and $28.1 \%$. Moreover, the peak value of $R C S$ reduction can reach $44.9 \mathrm{~dB}$. After testing the three reflecting screens in a microwave anechoic chamber, it was found that the measured results were basically in agreement with the simulation data, which verified the feasibility of the design. The design of reflecting screen proposed in this paper can provide a new method and approach for new ultra-wideband stealth technology.
\end{abstract}

\section{Keywords}

Phase gradient metasurface, ultra-wideband, low RCS

\section{Introduction}

In recent years, with the increasingly fierce competition in the military field, traditional stealth technology [1-11] has been unable to meet the current requirements of high performance and low interception. The emergence and development of Electromagnetic Material (EM) undoubtedly opens the door of new stealth technology. Left Handed Material (LHM) [12], [13], Electromagnetic Band Gap (EBG), Frequency Selective Surface (FSS), Artificial Magnetic Conductor (AMC), Phase Gradient Metasurface (PGM) [14-16] and other new EMs arise at the historic moment. Among them, PGM has attracted much attention because of its unique characteristics of abnormal refraction.
With the development of radar technology, new stealth technology is no longer satisfied with the reduction of RCS in a single frequency point or narrow band [17-20]. In order to realize ultra-wideband RCS reduction, in 2015, Chen Wengang et al. from the University of Surrey, Guildford, UK designed a surface covered with a checkerboard of alternating EBG structures, and resulted in a wider frequency band RCS reduction, which RCS reduction, compared to a PEC surface, of $10 \mathrm{~dB}$ can be realized over $60 \%$ frequency bandwidth [21]. In 2018, Su Jianxun's team of the Science and Technology on Electromagnetic Scattering Laboratory, Communication University of China, proposed a checkerboard metasurface based on a novel physical mechanism, optimized multielement phase cancellation (OMEPC) for greatly expanding the bandwidth of RCS reduction. The proposed metasurface not only can achieve $10 \mathrm{~dB}$ RCS reduction in a super-wide frequency band from 5.5 to $32.3 \mathrm{GHz}$ with a ratio bandwidth $\left(f_{\mathrm{H}} / f_{\mathrm{L}}\right)$ of $5.87: 1$ under normal incidence for both polarizations, but also has a good performance under wide-angle oblique incidences [22]. Similar to the checkerboard metasurface, another known method is where PGMs are designed based on generalized Snell-Descartes' law for the anomalous reflection.

Different from other EMs, PGM can introduce phase mutations at wavelength scales at different positions on the interface, so it no longer satisfies the classical law of reflection and refraction, but follows the generalized Snell's law. In 2011, Professor Yu from Harvard University proposed the PGM of the ' $\mathrm{V}$ ' shaped array structure for the first time. By changing the rotation angle of the element structure, the abnormal refraction of light waves was realized and the generalized Snell's law was verified [1]. In 2012, Professor Zhou from Lei's research group of Fudan University proposed PGM with an ' $\mathrm{H}$ ' shaped structure, which can efficiently couple incident electromagnetic waves into surface waves [4]. In 2013, Feng et al. from Nanjing University designed an active impedance PGM and introduced varactor diode into the resonant structure to realize $360^{\circ}$ dynamic regulation of the reflection phase of electromagnetic wave [23]. Using a rectangular Split Resonator Ring (SRR), Professor Qu from Shaobo's team of Air Force Engineering University changed the structural parameters of the resonator ring and realized the coupling of 
a specially polarized electromagnetic wave into a surface wave, thus effectively reducing RCS [3]. In 2017, Ge Chenchen of Huazhong Science and Technology University adopted PGM with a composite structure of two SRRs to realize the significant reduction of monostatic backward RCS in dual-frequency band [24].

In this paper, a reflective metasurface based on double diagonal opening cross petal SRR was designed. Based on this metasurface unit-cell, metal petals with six different geometric parameters were arranged periodically to form an ultra-wideband PGM and the same phase mutation was introduced at different locations of the metasurface to realize free regulation of the reflection phase of the incident electromagnetic wave. In the final design, the reflecting screens of the three new spiral configurations based on PGM achieved the reduction of RCS over $10 \mathrm{~dB}$ within the ultra-wide band with the average relative bandwidth of $30.5 \%$ and the maximum reduction of RCS up to $44.9 \mathrm{~dB}$ as scheduled, providing a new design method and approach for the new ultra-wideband stealth technology.

\section{Design of Phase Gradient Metasurface}

\subsection{Theoretical Model}

PGM completely follows the generalized Snell's law. By introducing phase mutation at different positions of the interface and forming a certain phase difference, the wave front of the electromagnetic wave can be changed to achieve the purpose of freely regulating the propagation of the electromagnetic wave.

As shown in Fig. 1, suppose that a beam of electromagnetic wave is incident at an $\theta_{\mathrm{i}}$ angle from point $\mathrm{A}$, after passing through the reflecting surface, it is emitted at an $\theta_{\mathrm{r}}$ angle to point $\mathrm{C}$. The phase gradient of unit length on the reflecting surface is $\xi$, and then:

$$
\begin{gathered}
\sin \theta_{\mathrm{r}}-\sin \theta_{\mathrm{i}}=\frac{\lambda}{2 \pi n_{\mathrm{i}}} \xi, \\
\theta_{\mathrm{r}}=\arcsin \left(\frac{\lambda}{2 \pi n_{\mathrm{i}}}+\sin \theta_{\mathrm{i}}\right) .
\end{gathered}
$$

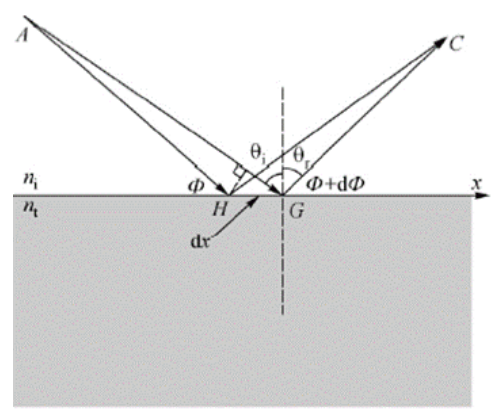

Fig. 1. Schematic diagram of Generalized Snell's law of reflection.
Generally, the phase gradient is realized by a two-dimensional array formed by the arrangement of $n$ metasurface elements, and the phase shift corresponding to one element is $\Delta \phi=2 \pi / n$. The element size along the gradient direction is set as $p$, and the phase gradient can be obtained as follows:

$$
\xi=\frac{\Delta \phi}{p}=\frac{2 \pi}{n p} .
$$

Combined with formula (2) and (3), the reflected angle $\theta_{\mathrm{r}}$ can be calculated:

$$
\theta_{\mathrm{r}}=\arcsin \left(\frac{\lambda}{2 \pi n_{\mathrm{i}}} \frac{\Delta \phi}{p}+\sin \theta_{\mathrm{i}}\right)=\arcsin \left(\frac{\lambda}{n_{\mathrm{i}} \cdot n p}+\sin \theta_{\mathrm{i}}\right)
$$

When the electromagnetic wave is perpendicularly incident, the reflection angle is:

$$
\theta_{\mathrm{r}}=\arcsin \left(\frac{\lambda}{n_{\mathrm{i}} \cdot n p}\right)
$$

1) If $0<\theta_{\mathrm{r}}<1$, then the reflected wave turns into abnormal reflection. Therefore, PGMs fall into this category.

2) If $\theta_{\mathrm{r}}>1$, then the incident wave is bound to the surface of the material, and the surface wave coupling is realized. So, the checkerboard metasurfaces fall into this category.

Assuming that the metasurface elements are arranged along the $\mathrm{x}$ and $\mathrm{y}$ axis respectively to form a two-dimensional metasurface, according to the generalized Snell's law of reflection, it can be seen that the two-dimensional metasurface satisfies:

$$
\begin{gathered}
\sin \theta_{\mathrm{r}}-\sin \theta_{\mathrm{i}}=\frac{\lambda}{2 \pi n_{\mathrm{i}}} \sqrt{(\mathrm{d} \phi / \mathrm{d} x)^{2}+(\mathrm{d} \phi / \mathrm{d} y)^{2}} \\
\tan \varphi_{\mathrm{r}}=\frac{\mathrm{d} \phi / \mathrm{d} y}{\mathrm{~d} \phi / \mathrm{d} x} .
\end{gathered}
$$

In the formula, $\theta_{\mathrm{r}}$ and $\varphi_{\mathrm{r}}$ are used to represent the pitch angle and azimuth angle of the reflected electromagnetic wave. In order to realize UWB singular reflection, the phase gradient $\xi=\mathrm{d} \phi / p$ should be less affected by frequency, that is, the phase shift between adjacent elements $\Delta \phi=2 \pi / n$ should remain unchanged in the designed frequency band.

\subsection{Design of Metasurface Unit-Cell}

In this paper, based on the classical cross structure [15] and SRR, a double diagonal opening cross petal structure unit was designed, as shown in Fig. 2. The whole structure was composed of three layers, the unit period was $p=$ $10 \mathrm{~mm}$, the middle layer was F4B $\left(\varepsilon_{\mathrm{r}}=2.65, \tan \delta=0.001\right)$ medium substrate with thickness $h=3 \mathrm{~mm}$, and the bottom layer was metal floor with thickness $t=0.036 \mathrm{~mm}$. The metal structure was designed on the surface of the medium 


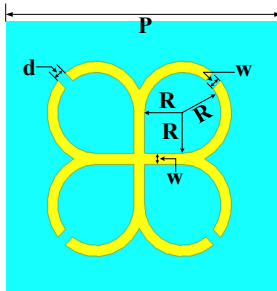

(a)

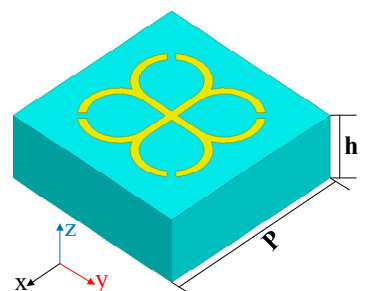

(b)
Fig. 2. The structure of double diagonal opening cross petal type metasurface unit-cell: (a) vertical view and (b) lateral view.

plate. The 'cross petal' was composed of an equal-length, vertically intersecting 'cross' and four three-quarters of the circle tangent to each other. The opening was located at two diagonal positions of the pattern, the petal radius was $R$, the petal width was $w=0.4 \mathrm{~mm}$, and the opening gap was $d=0.4 \mathrm{~mm}$. Compared to other similar structures, the proposed metasurface unit-cell combines the strengths of the classical cross structure and SRR, and has the outstanding advantages of small size, high linearity and wide band.

When electromagnetic waves are perpendicularly incident, the polarization electric field of $x$ and $y$ will generate induced current on the "cross petal" patch parallel to the polarization direction. The coupling between the metal floor and the upper structure causes the metal floor to produce a reverse current. Displacement current is generated in the dielectric plate. The induced current and the displacement current eventually form a closed loop and produce magnetic resonance. The coupling between the upper metal structure and the floor can generate a number of different local magnetic resonance circuits with different frequencies, that is, a number of magnetic resonance modes. By using the idea of multi-mode cascade and adjusting the structure of the element, the linearity and range of phase change can be improved, so as to widen the working bandwidth of the element. In order to realize UWB singular reflection, the phase difference between adjacent units at the central frequency $f=14 \mathrm{GHz}$ is guaranteed to be basically unchanged at $\Delta \phi=60^{\circ}$. After optimization design, $R=0.74 \mathrm{~mm}, 0.8 \mathrm{~mm}, 0.89 \mathrm{~mm}, 1.09 \mathrm{~mm}, 1.31 \mathrm{~mm}$ and $1.46 \mathrm{~mm}$ are respectively adopted in this paper. Figure 3 shows the frequency response of the metasurface unit-cell at different $R$ values.

It can be seen from Fig. 3(a) that the reflectivity of the six metasurface unit-cells of different sizes is above $97 \%$, indicating that all the six units work in the state of total reflection. As shown in Fig. 3(b) and Tab. 1, when $R=0.74 \mathrm{~mm}, 0.8 \mathrm{~mm}, 0.89 \mathrm{~mm}, 1.09 \mathrm{~mm}, 1.31 \mathrm{~mm}$ and $1.46 \mathrm{~mm}$, the reflection phases at $14 \mathrm{GHz}$ are $-116^{\circ},-175^{\circ}$, $-237^{\circ},-297^{\circ},-356^{\circ}$ and $-414^{\circ}$ respectively, the adjacent phase intervals are about $60^{\circ}$ and the reflection phase curve has a good linearity within $8-20 \mathrm{GHz}$, maintaining a relatively stable phase difference, satisfying the condition for realizing ultra-wideband abnormal reflection.

The six metasurface unit-cells of different sizes were arranged along the $y$ direction and periodic extension was carried out along the $x$ direction. The one-dimensional metasurface unit-cell is shown in Fig. 4(a). The six metasurface elements of different sizes were arranged along the $x$ and $y$ directions respectively and the phase gradient components in the two directions were equal. The two-dimensional gradient metasurface is shown in Fig. 4(b).

Figure 5 shows the RCS values of 1-D and 2-D PGM elements. The RCS of the one-dimensional PGM is lower than $-10 \mathrm{dBsm}$ in the frequency band from $8.3 \mathrm{GHz}$ to 15.3 GHz and reaches the minimum RCS at $11.2 \mathrm{GHz}$. The

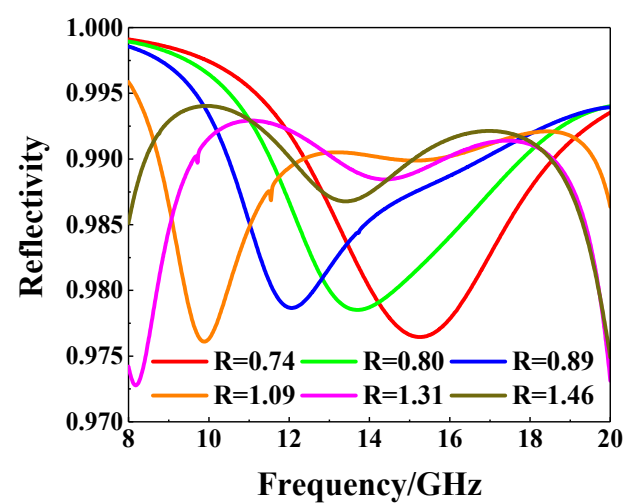

(a)

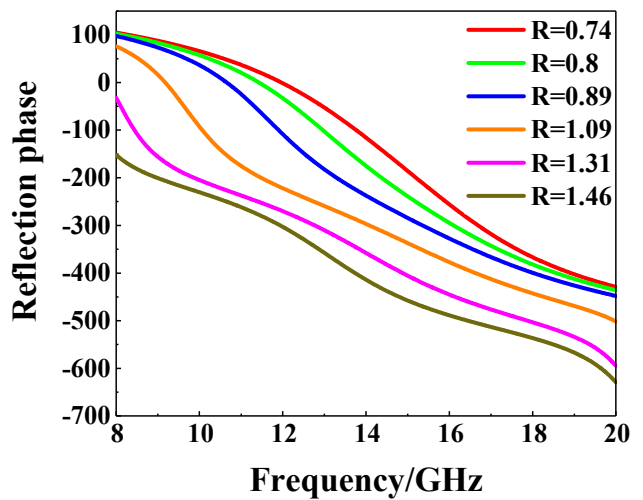

(b)

Fig. 3. Frequency response at different $R$ value: (a) reflectivity and (b) reflection phase.

\begin{tabular}{|c|c|c|c|}
\hline Num & $\mathbf{R}[\mathbf{m m}]$ & Reflectivity[\%] $^{\text {[oflection }}$ phase $^{\circ}{ }^{\circ}{ }^{\text {] }}$ \\
\hline 1 & 0.74 & 98.02 & -115.69 \\
\hline 2 & 0.8 & 97.87 & -175.04 \\
\hline 3 & 0.89 & 98.53 & -237.39 \\
\hline 4 & 1.09 & 99.03 & -296.89 \\
\hline 5 & 1.31 & 98.86 & -357.71 \\
\hline 6 & 1.46 & 98.74 & -413. \\
\hline
\end{tabular}

Tab. 1. Frequency response at different $R$ value.

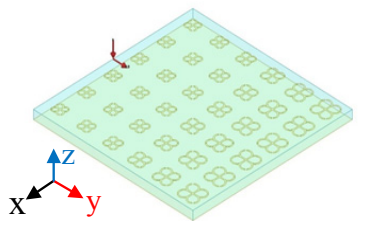

(a)

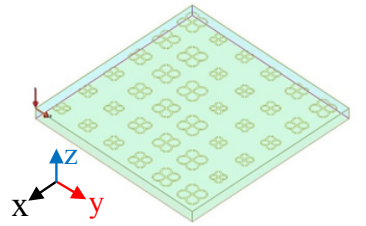

(b)
Fig. 4. (a) One-dimensional and (b) two-dimensional PGM elements. 


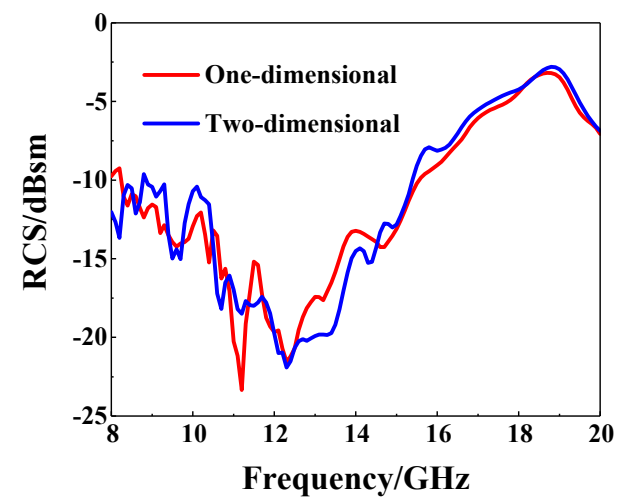

Fig. 5. RCS comparison between one and two-dimensional PGM elements.

RCS of two-dimensional phase-gradient metasurface is lower than $-10 \mathrm{dBsm}$ in the frequency range of $8-15.3 \mathrm{GHz}$ and reaches the minimum value at $12.3 \mathrm{GHz}$. Due to the fact that six "cross petal" type metasurface unit-cells of different sizes do not strictly meet the phase difference at the central frequency of $14 \mathrm{GHz}$ and the coupling resonance between each metasurface is not uniform due to the influence of element spacing, the frequency point of the minimum RCS moves towards the direction of low frequency. Figure 6 shows the two-dimensional and threedimensional scattering patterns when the electromagnetic wave is perpendicular to the metasurface (the direction of the electric field is parallel to the $\mathrm{Y}$-axis).

According to (5), the theoretical value of the reflection angle $\theta_{\mathrm{r}}$ of the reflected electromagnetic wave of the one-dimensional PGM unit-cell at the frequency point of $11.2 \mathrm{GHz}$ (that is the RCS reaches the minimum) is $26.51^{\circ}$. Similarly, according to (6), the azimuth angle $\varphi_{\mathrm{r}}$ and pitch angle $\theta_{\mathrm{r}}$ of the reflected electromagnetic wave of the twodimensional PGM unit-cell at the frequency point $12.3 \mathrm{GHz}$ (that is the RCS reaches the minimum) can be calculated as $45^{\circ}$ and $35.09^{\circ}$.

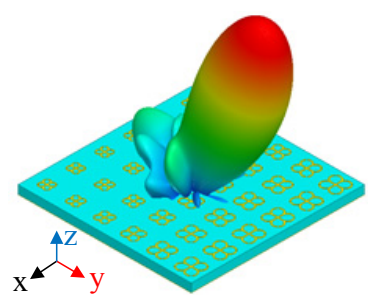

(a)

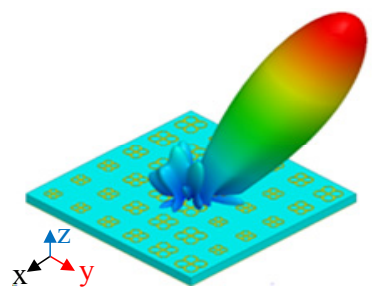

(c)

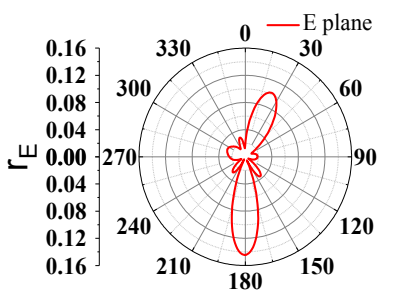

(b)

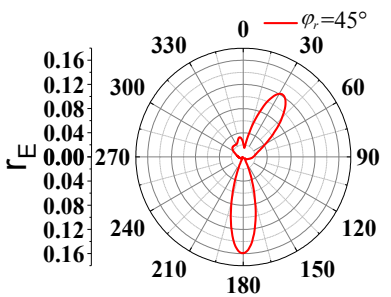

(d)
Fig. 6. 2-D and 3-D scattering patterns when electromagnetic waves are perpendicularly incident: (a) 3-D scattering pattern of one-dimensional PGM element, (b) 2-D pattern of E plane, (c) 3-D scattering pattern of one-dimensional PGM element and (d) 2-D pattern of $\varphi_{\mathrm{r}}=45^{\circ}$.

\begin{tabular}{|c|c|c|c|}
\hline & 1-D & \multicolumn{2}{|c|}{ 2-D } \\
\cline { 2 - 4 } & $\theta_{\mathrm{r}}\left({ }^{\circ}\right)$ & $\theta_{\mathrm{r}}\left({ }^{\circ}\right)$ & $\varphi_{\mathrm{r}}\left({ }^{\circ}\right)$ \\
\hline Theoretical value & 26.51 & 35.09 & 45 \\
\hline Actual value & 23 & 32 & 45 \\
\hline
\end{tabular}

Tab. 2. The comparison between the theoretical value and the actual value of the reflected electromagnetic wave angle.

It can be seen from Tab. 2 that the theoretical value of the reflected electromagnetic wave angle is in high agreement with the actual value, which verifies the correctness of the generalized Snell's law. One-dimension and twodimensional PGM elements realize the free control of the angle of the reflected electromagnetic wave, which can be used to realize the design of antenna reflecting screen with low RCS.

\section{Simulation and Experimental Verification}

\subsection{Simulation and Analysis of Results}

In order to make the energy of the scattering beam more evenly dispersed to achieve stability at large incident angle, this paper chose a new spiral structure instead of traditional arrangements of arrays. As shown in Fig. 7 below, rotate and number the 1-D and 2-D PGM in Fig. 6.

It can be seen from the propagation direction of reflected electromagnetic waves in Fig. 7 that the phases of elements $1,3,5$ and 7 are $\pi / 2$ for each interval, and the phases of elements $2,4,6$ and 8 are also $\pi / 2$ for each interval. The phase intervals of units $1,2,3,4,5,6,7$ and 8 are $\pi / 4$ by combining one and two dimensional metasurface elements. In terms of arrangement, the $6 \times 6$ structure of basic unit above covers the phase range of $360^{\circ}$ regardless of whether the 1-D and 2-D metasurface elements are separated or used together. In this paper, these 8 metasurface elements were arranged according to the arrangement mode as shown in Fig. 8, and three models of reflecting screen with the size of $240 \mathrm{~mm} \times 240 \mathrm{~mm}$ were finally designed.

In order to verify the designed reflecting screen, HFSS 14.0 was used to conduct full-wave simulation of the reflecting screen. The incident wave was incident vertically along the $x$ direction to excite the metasurface. In order to obtain the reduction effect of ultra-wideband RCS, this paper collates the simulated RCS data every $0.1 \mathrm{GHz}$ by comparing the metal plate of the same size, as shown in Fig. 9.

As can be seen from Fig. 9(a), the reduction of RCS in model 1 is much larger than that in model 2 and model 3, which show a relatively gentle reduction. The RCS reduction peaks of the three models at their respective resonance points $(13.6 \mathrm{GHz}, 12.1 \mathrm{GHz}, 11.4 \mathrm{GHz})$ are $44.9 \mathrm{~dB}, 15.6 \mathrm{~dB}$, 


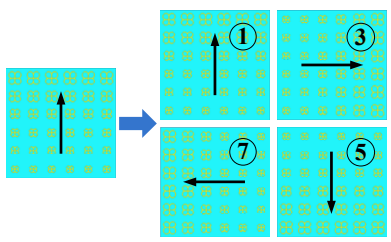

(a)

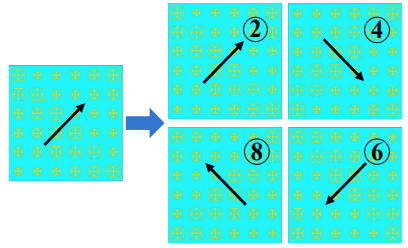

(b)
Fig. 7. $6 \times 6$ arrangement of metasurface elements: (a) onedimension and (b) two-dimension.

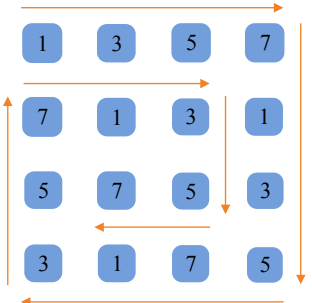

(a)
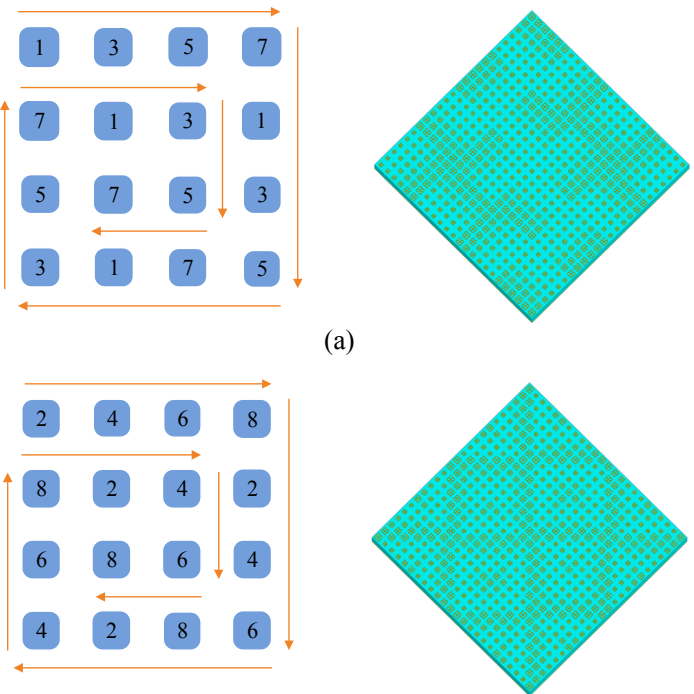

(b)

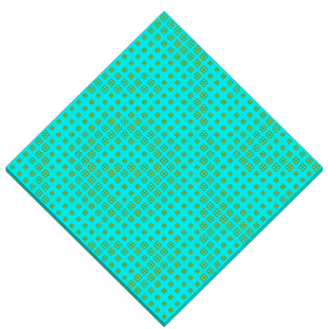

(c)

Fig. 8. Schematic diagram of different arrangement of basic unit and model of reflection screen: (a) model 1, (b) model 2 and (c) model 3.

$17.2 \mathrm{~dB}$ respectively, and the relative bandwidth of the RCS reduction reaching above $10 \mathrm{~dB}$ are $34.5 \%, 28.8 \%$, $28.1 \%$, respectively. In general, the reduction trend of RCS of the three models is the same. The average relative bandwidth of the reduction of RCS is $30.5 \%$, which meets the requirements of ultra-wideband. Figure 9(b), (c) and (d) show the bistatic RCS of model 1, 2 and 3 at their respective resonant frequency points. It can be clearly seen that the three models have an obvious RCS reduction effect when the electromagnetic wave is vertically incident. Model 1 has the most significant reduction effect in the $\pm 5^{\circ}$ angular domain, but the disadvantage is that the angular domain is too narrow and the scattering energy is not evenly distributed. The bistatic RCS of model 2 and model 3 in the $\pm 15^{\circ}$ angular domain are all smaller than the metal plate, and the scattered energy distribution is more even than that of model 1, with significant diffuse reflection effect.

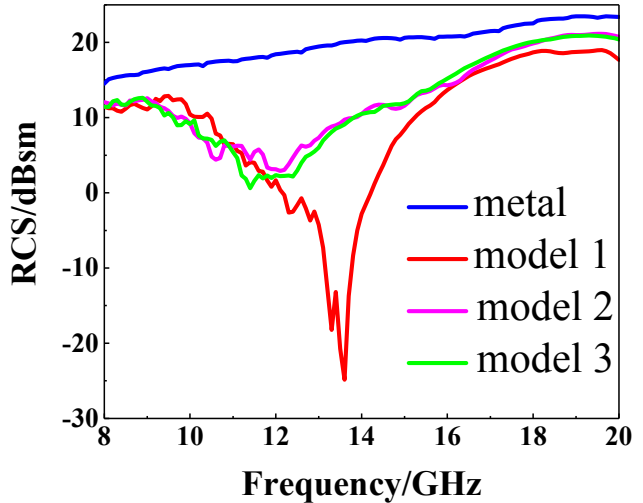

(a)

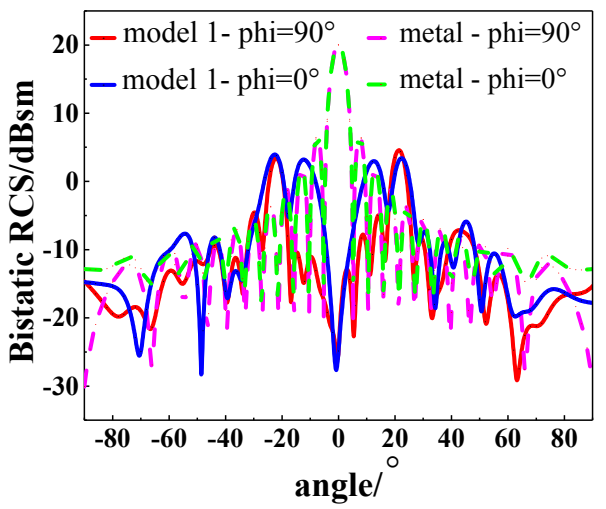

(b)

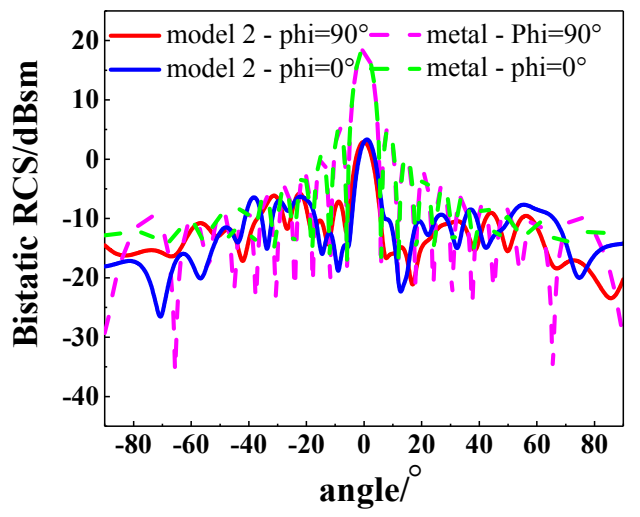

(c)

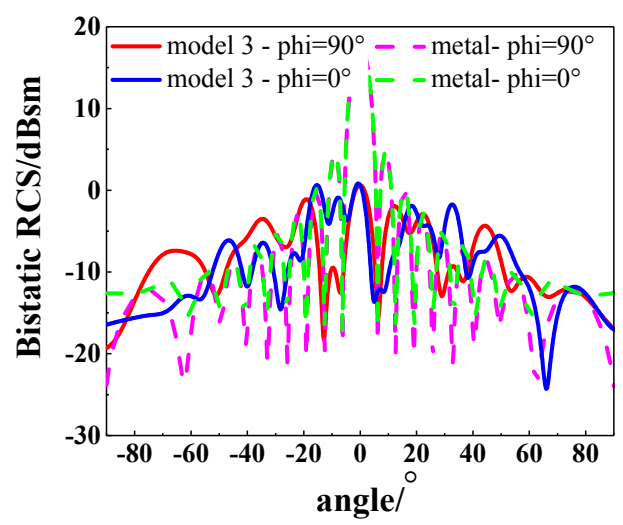

(d)

Fig. 9. Simulation of monostatic and bistatic RCS: (a) monostatic RCS, (b) bistatic RCS of model 1, (c) bistatic RCS of model 2 and (d) bistatic RCS of model 3. 
In this paper, the RCS reduction characteristics of the designed reflecting screen were further analyzed, and the three-dimensional pattern of electric field energy and twodimensional scattering pattern of $\mathrm{E}$ plane and $\mathrm{H}$ plane of each model when the electromagnetic wave was vertically incident were obtained through simulation as shown in Fig. 10. According to the law of conservation of energy, the increase of scattering energy of side lobes can effectively inhibit the energy of main lobe, thus realizing the RCS reduction in the vertical direction. As can be seen from the pattern, when the electromagnetic wave is incident vertically, the metal plate has a strong main lobe in the vertical direction. Figures 10(a), (b) and (c) clearly show that these three models of reflecting screen based on PGM have obvious inhibition effect on the main lobe. Model 1 has the best inhibition effect on the main lobe, while Model 2 has a more uniform scattering, higher symmetry and stronger scattering ability.

By comparing and analyzing the performance of RCS reduction of three models, the model 1 with the best performance is selected for further analysis. Figure 11(a) shows the RCS reduction comparison of model 1 under different incident wave conditions of $\mathrm{x}$ polarization and $\mathrm{y}$ polarization. It can be seen from the figure that, under the condition of two polarizations, the reduction trend of RCS is consistent and the curves are roughly coincident, reflecting the polarization insensitivity of the low scattering characteristic of this reflecting model. But model 1 has stronger scattering ability for y polarization incident wave near the resonance point. Next, the performance of RCS reduction under oblique incidence is studied. Figure 11(b) shows the comparison results of RCS reduction when y polarization waves are incident at $0^{\circ}, 30^{\circ}$ and $45^{\circ}$, respectively. It can be seen that the reflecting screen still has ultra-wideband
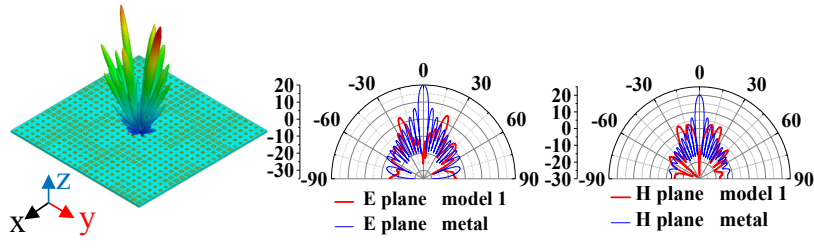

(a)
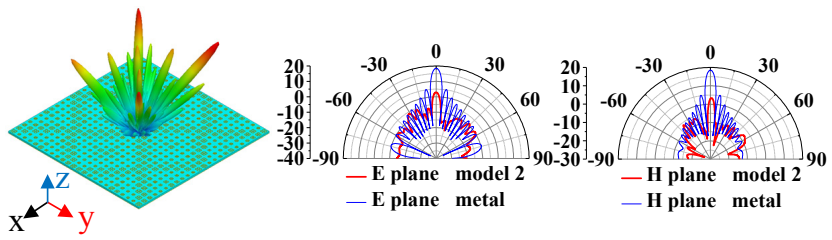

(b)
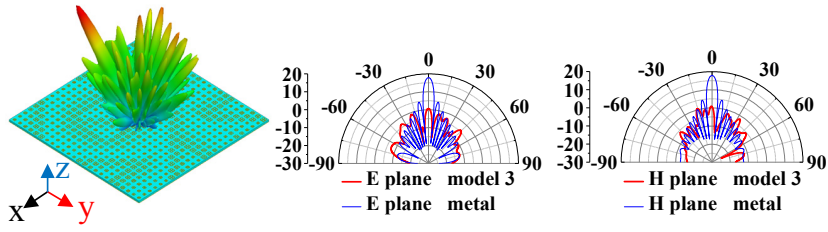

(c)

Fig. 10. Three dimensional pattern of electric field energy and two dimensional pattern of $\mathrm{E}$ plane and $\mathrm{H}$ plane: (a) model 1, (b) model 2 and (c) model 3.

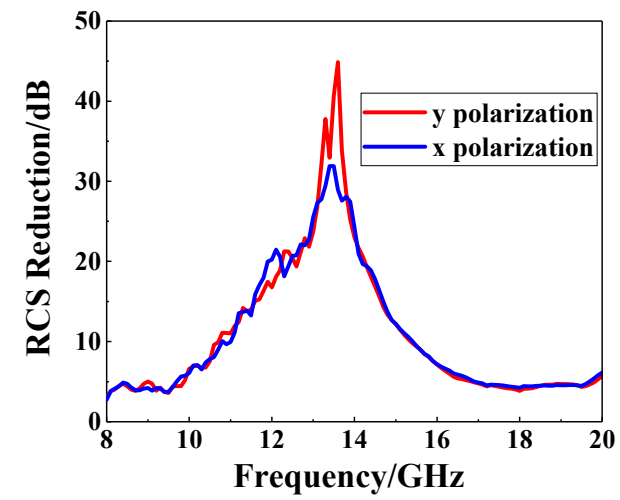

(a)

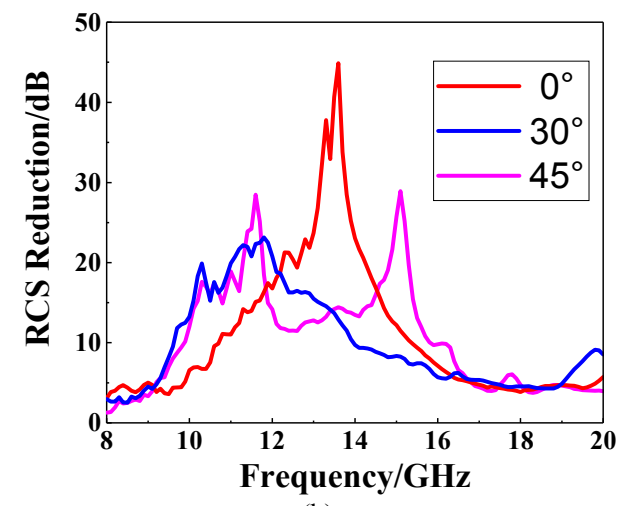

(b)

Fig. 11. Monostatic RCS reduction: (a) $x$ polarization and y polarization and (b) incidence at different angles.

\begin{tabular}{|c|c|c|c|c|c|}
\hline \multirow{2}{*}{ Article } & \multirow{2}{*}{$\begin{array}{c}\sigma_{\mathrm{R}} \\
(\mathrm{dB})\end{array}$} & OFB $(\mathrm{GHz})$ & \multicolumn{2}{|c|}{$\mathrm{RBW}(\%)$} & \multirow{2}{*}{$\begin{array}{c}\text { Peak value } \\
(\mathrm{dB})\end{array}$} \\
\cline { 4 - 5 } & & $0^{\circ}$ & Other angle & \\
\hline$[21]$ & 10 & $4.1-7.9$ & 63 & $34\left(40^{\circ}\right)$ & 30 \\
\hline$[22]$ & 10 & $5.5-32.3$ & 141.8 & $108\left(40^{\circ}\right)$ & 21.3 \\
\hline$[24]$ & 10 & $7.8,9.4$ & $/$ & $/$ & 25 \\
\hline This work & 10 & $10.8-15.3$ & 34.5 & $45.9\left(45^{\circ}\right)$ & 44.9 \\
\hline
\end{tabular}

Tab. 3. Comparison of our work and previous researches. $\sigma_{\mathrm{R}}$ : RCS reduction; OFB: Operating frequency band; RBW: Relative bandwidth.

RCS reduction characteristics at 30 and 45 oblique incidences, even when RCS reduces to $-10 \mathrm{~dB}$ at 45 oblique incidence, the frequency band range is wider. The performance of RCS reduction is improved at $9.2 \mathrm{GHz}-11.7 \mathrm{GHz}$ and $14.6 \mathrm{GHz}-16.5 \mathrm{GHz}$, but the reduction performance is worse near the resonance point $(12.1 \mathrm{GHz}-14.5 \mathrm{GHz})$, indicating that the designed reflecting screen in this paper has stability at large incident angle.

By comparing and analyzing the traditional checkerboard metasurfaces, coding diffusion metasurfaces based on optimization techniques and other PGMs, as shown in Tab. 3, obviously, it can be seen that our work has an overwhelming advantage in stability at large incident angle. In addition, the proposed PGM has the maximum RCS reduction peak in the operating band, in other words, its performance of the backward RCS reduction is the best. 


\subsection{Experimental Verification}

In order to verify the designed reflecting screen based on PGM, according to the above simulated structural parameters of metasurface, the test sample of the reflecting screen with a size of $240 \mathrm{~mm} \times 240 \mathrm{~mm}$ was processed using printed circuit board technology, as shown in Fig. 12. F4B substrate with thickness $h=3 \mathrm{~mm}$ was used for the dielectric plate, and the metal surface was treated with heavy tin processing to prevent metal oxidation from affecting the experimental results. The whole test process was carried out in microwave anechoic chamber using free space method.

Fix the test sample vertically in the center of the rotating foam tower. Two standard horn antennas for sending and receiving electromagnetic waves were fixed on a bracket directly in front of the foam tower, which were connected to two ports of the Agilent N5230C vector network analyzer respectively. The horn openings shall be aligned with the geometric center of the sample, and the

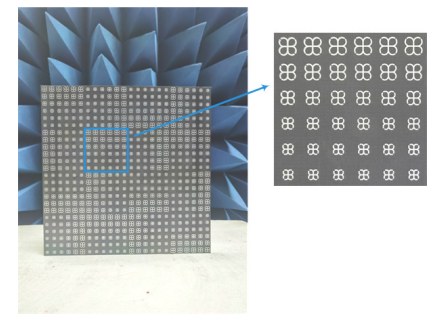

Fig. 12. The experimental sample.

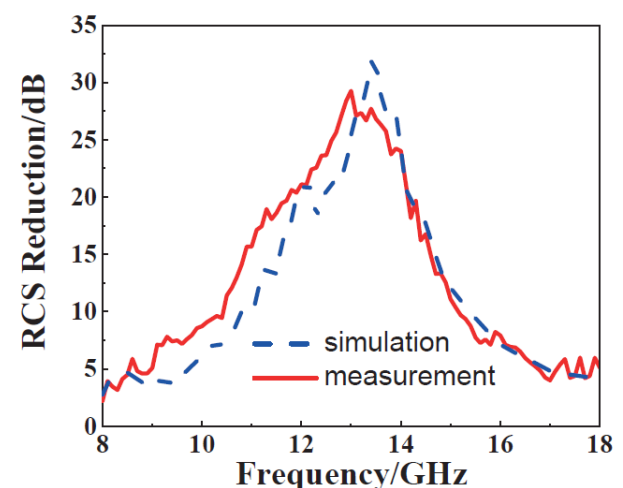

(a)

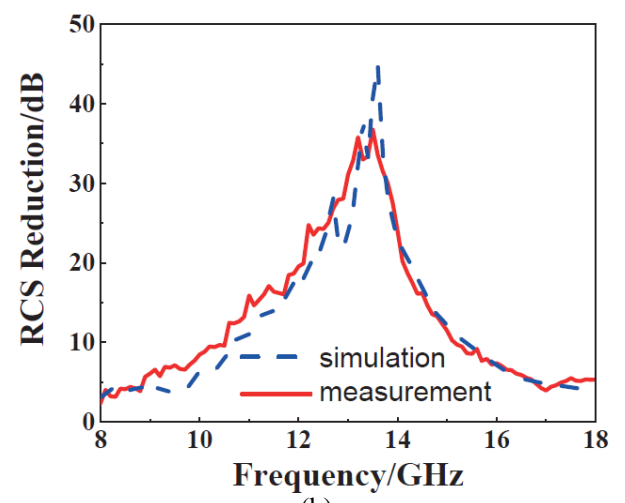

(b)

Fig. 13. The measured and simulated curve of RCS reduction: (a) x polarization and (c) y polarization. connecting angle between the two horn openings and the center of the sample shall be less than $5^{\circ}$ when placed. In order to ensure that the incident electromagnetic wave is a plane wave, the distance between the horn antenna and the test sample shall meet the far field condition $\left(2 D^{2} / \lambda\right)$, and the distance set in this paper was $3 \mathrm{~m}$. The curve of RCS reduction was obtained by testing the metal plate and the designed reflector with the same size as the test sample.

Figure 13 shows the measured and simulated curve of the sample's RCS reduction, which can be clearly seen: in the full frequency band, RCS reduction effect of sample is significant, and the range of frequency band with RCS reduction greater than $10 \mathrm{~dB}$ is broadened. In the low frequency band, the measured effect of RCS reduction is better, and in the high frequency band, the measured curve coincides with the simulation curve, which indicates the consistency between the test result and the simulation result. It can also be seen from the measured results that the peak value of RCS reduction is reduced compared with that of the simulation. This is because the two horns are too close to each other during the test, and part of the electromagnetic wave radiated by the transmitting antenna will directly diffract from the edge of the horns into the receiving antenna without reflection, causing clutter to affect the results. In general, the test results show that the designed reflecting screen based on PGM has high performance in $\mathrm{RCS}$ reduction.

\section{Conclusion}

The ultra-wideband low RCS reflecting screen based on PGM designed in this paper adopts the reflecting metasurface unit-cell with metal structure of double diagonal opening cross petal, whose reflectivity is over $97 \%$ in the range of 8-20 GHz frequency band. By changing the structure size of the element, the reflection phase of the metasurface element can be controlled freely, and the reflectivity basically remains unchanged. One and two dimensional PGM were designed with six different size units, which can freely adjust and control the reflection angle of the electromagnetic wave directly incident. Based on this, three new spiral progressive layout UWB reflecting screen were designed, and the high efficiency of RCS reduction was verified by the far field simulation results. The relative bandwidth of RCS reduction over $10 \mathrm{~dB}$ was $34.5 \%, 28.8 \%$ and $28.1 \%$ respectively, and the peak value of RCS reduction can reach $44.9 \mathrm{~dB}$. The reflecting screen was tested in the anechoic chamber and the measured results were basically consistent with the simulation data, which verified the feasibility of the design and provided a new design method and approach for the new UWB stealth technology.

\section{Acknowledgements}

National Natural Science Foundation of China under grant (Nos. 61671464, 61701523 and 61801508); Natural 
Science Basic Research Program of Shaanxi Province, China (Grant No. 2019JQ-103); General Foundation of China Postdoctoral Science Foundation (Grant No. 2019M653960); Supported by the Postdoctoral Innovative Talents Support Program of China (Grant No. BX20180375) and Supported by the Yong Talents Support Program of Shanxi Province, China (Grant No. 20200108)

\section{References}

[1] YU, N. F., GENEVET, P., KATS, M. A., et al. Light propagation with phase discontinuities: Generalized laws of reflection and refraction. Science, 2011, vol. 334, no. 6054, p. 333-337. DOI: $10.1126 /$ science. 1210713

[2] SUN, S. L., YANG, K. Y., WANG, C. M., et al. High-efficiency broadband anomalous reflection by gradient meta-surfaces. Nano Letters, 2012, vol. 12, no. 12, p. 6223-6229. DOI: $10.1021 / \mathrm{n} 13032668$

[3] WANG, J. F., QU, S. B., MA, H., et al. High-efficiency spoof plasmon polariton coupler mediated by gradient metasurfaces. Applied Physics Letters, 2012, vol. 101, no. 20, p. 1-4. DOI: $10.1063 / 1.4767219$

[4] SUN, S. L., HE, Q., XIAO, S. Y., et al. Gradient-index metasurfaces as a bridge linking propagating waves and surface waves. Nature Materials, 2012, vol. 11, p. 426-431. DOI: $10.1038 /$ nmat3292

[5] LI, Y. F., ZHANG, J. Q., QU, S. B., et al. Wideband radar cross section reduction using two-dimensional phase gradient metasurfaces. Applied Physics Letters, 2014, vol. 104, no. 22 , p. 1-5. DOI: $10.1063 / 1.4881935$

[6] SHI, H. Y., LI, J. X., ZHANG, A. X., et al. Gradient metasurface with both polarization-controlled directional surface wave coupling and anomalous reflection. IEEE Antennas and Wireless Propagation Letters, 2015, vol. 14, p. 104-107. DOI: 10.1109/LAWP.2014.2356483

[7] ZHUANG, Y. Q., WANG, G. M., ZHANG, C. X., et al. Design and experimental verification of single-layer high-efficiency transmissive phase-gradient metasurface. Acta Physica Sinica, 2016, vol. 65, no. 15, p. 1-6. (In Chinese) DOI: 10.7498/aps.65.154101

[8] ZHUANG, Y. Q., WANG, G. M., ZHANG, X. K., et al. Design of reflective linear-circular polarization converter based on phase gradient metasurface. Acta Physica Sinica, 2016, vol. 65, no. 15, p. 1-6. (In Chinese) DOI: 10.7498/aps.65.154102

[9] FAN, Y., QU, S. B., WANG, J. F., et al. Broadband anomalous reflector based on cross-polarized version phase gradient metasurface. Acta Physica Sinica, 2015, vol. 64, no. 18, p. 1-6. (In Chinese) DOI: 10.7498/aps.64.184101

[10] ZHENG, Q. Q., LI, Y. F., ZHANG, J. Q., et al. Wideband, wideangle coding phase gradient metasurfaces based on PancharatnamBerry phase. Scientific Reports, 2017, vol. 7, p. 1-13. DOI: 10.1038/srep43543

[11] GONG, S. X., LIU, Y., ZHANG, P. F., et al. Prediction and Reduction of Antenna Radar Cross Section. Xi'an(China): Xi'dian University Press, 2010

[12] PENDRY, J. B., HOLDEN, A. J., STEWART, W. J., et al. Extremely low frequency plasmons in metallic mesostructures. Physical Review Letters, 1996, vol. 76, no. 25, p. 4773-4776. DOI: 10.1103/PhysRevLett.76.4773

[13] PENDRY, J. B., HOLDEN, A. J., ROBBINS, D. J., et al. Magnetism from conductors and enhanced nonlinear phenomena.
IEEE Transactions on Microwave Theory and Techniques, 1999, vol. 47, no. 11, p. 2075-2084. DOI: $10.1109 / 22.798002$

[14] HOLLOWAY, C. L., KUESTER, E. F., GORDON, J. A., et al. An overview of the theory and applications of metasurfaces: The two-dimensional equivalents of metamaterials. IEEE Antennas and Propagation Magazine, 2012, vol. 54, no. 2, p. 10-35. DOI: 10.1109/MAP.2012.6230714

[15] WU, C. J., CHENG, Y. Z., WANG, W. Y., et al. Design and radar cross section reduction experimental verification of phase gradient meta-surface based on cruciform structure. Acta Physica Sinica, 2015, vol. 64, no. 16, p. 1-4. (In Chinese) DOI: 10.7498/aps.64.164102

[16] LI, T. J., LIANG, J. G., LI, H. P., et al. Ultra-thin single-layer transparent geometrical phase gradient metasurface and its application to high-gain circularly-polarized lens antenna. Chinese Physics B, 2016, vol. 25, no. 9, p. 1-5. DOI: 10.1088/1674$1056 / 25 / 9 / 094101$

[17] GUO, W. L., WANG, G. M., LI, H. P., et al. A novel broad gradient metasurface. Journal of Microwaves, 2016, vol. 32, no. 3, p. 51-59. (In Chinese) DOI: 10.14183/j.cnki.10056122.201603012

[18] LI, C., CHEN, M. Design of a ultra-wideband reflective phase gradient metasurface. Research \& Progress of SSE, 2020, vol. 40, no. $2, \quad$ p. 127-130. (In Chinese) DOI: 10.19623/j.cnki.rpsse.2020.02.010

[19] ZHANG, W. B., LIU, Y., GONG, S. X., et al. Wideband RCS reduction of a slot array antenna using phase gradient metasurface. IEEE Antennas and Wireless Propagation Letters, 2018, vol. 17, no. 12 , p. 2193-2197. DOI: 10.1109/LAWP.2018.2870863

[20] LIANG, J. J., HUANG, G. L., ZHAO, J. N., et al. Wideband phase-gradient metasurface antenna with focused beams. IEEE Access, 2019, vol. 7, p. 20767-20772. DOI: 10.1109/ACCESS.2019.2898550

[21] CHEN, W. G., BALANIS, C. A., BIRTCHER, C. R. Checkerboard EBG surfaces for wideband radar cross section reduction. IEEE Transactions on Antennas and Propagation, 2015, vol. 63, no. 6, p. 2636-2645. DOI: 10.1109/TAP.2015.2414440

[22] SU, J. X., LU, Y., LIU, J. Y., et al. A novel checkerboard metasurface based on optimized multielement phase cancellation for super-wideband RCS reduction. IEEE Transactions on Antennas and Propagation, 2018, vol. 66, no. 12, p. 7091-7099. DOI: 10.1109/TAP.2018.2870372

[23] ZHU, B. O., ZHAO, J. M., FENG, Y. J. Active impedance metasurface with full $360^{\circ}$ reflection phase tuning. Scientific Reports, 2013, vol. 3, p. 1-6. DOI: 10.1038/srep03059

[24] GE, C. C., CHENG, Y. Z., WANG, X., et al. Design and radar cross section reduction experimental verification of a double band phase gradient meta-surface. Journal of Microwaves, 2017, vol. 33 , no. 1 , p. 36-40. (In Chinese) DOI: 10.14183/j.cnki.10056122.201701008

\section{About the Authors...}

Kefeng JI was born in Jiangsu province, China. He received his B.S. degree from the Air Force Engineering University in 2019. He currently works towards his M.S. degree. In his research, he specializes in metamaterial, antenna design and RCS reduction techniques.

Jun GAO received the M.S. and Ph.D. degrees from the Air Force Missile Institute in 1984 and 1987, respectively. He joined the Air Force Missile Institute in 1987 as an as- 
sistant teacher. He became an associate professor in 2000. $\mathrm{He}$ is currently a professor of the Information and Navigation College, Air Force Engineering University of CPLA. He has authored and coauthored more than 100 technical journal articles and conference papers, and holds one China soft patent. His research interests include smart antennas, electromagnetic metamaterial and their antenna applications.

Xiangyu CAO received her M.S. degree from the Air Force Missile Institute in 1989. In the same year, she joined the Air Force Missile Institute. She received her Ph.D. degree in the Missile Institute of AFEU in 1999. From 1999 to 2002, she was engaged in postdoctoral research in Xidian University, China. She was a Senior Research Associate in the Department of Electronic Engineering, City University of Hong Kong from June 2002 to Dec. 2003. She is currently a professor and a senior member of IEEE. Her research interests include computational electromagnetic, electromagnetic metamaterials and their antenna applications.

Jiangfeng HAN received the B.S. and M.S. degree from the Information and Navigation Institute, Air Force Engineering University of CPLA, China, in 2012 and 2014, respectively. His research interest is in electromagnetic metamaterials and their antenna applications.

Huanhuan YANG has received the M. Eng. degree in Electronic Science and Technology from the Air Force Engineering University, Xi' an China, in 2012. His research activities focus in polarized reconfigurable antenna. He has authored and coauthored more than 30 scientific papers in major journals and international conferences. 